\title{
Envelopes and Geometrical Covers of Side-Payment Games and their Market Representations*
}

\author{
Philippe Artzner; Claude d'Aspremont ${ }^{\ddagger}$ and Louis-André Gérard-Varet ${ }^{\S}$
}

Received September 27, 1982; revised July 5, 1984

\begin{abstract}
This paper deals with issues concerning the core as a solution concept for games in coalitional form as well as the use of these games in representing economies of a certain formal type. Side-payment games are imbedded in the more general class of no-side-payment games. It is shown that to a given side-payment game having an empty core one may associate two different no-side payment games with the same (nonempty) core: the "envelope" and the "geometrical cover". The discrepancy is explained in terms of market games.
\end{abstract}

Key-words: Side-payment games, core, market games

AMS 1980 subject classification: Primary: 90D12

IAOR 1973 subject classification: Main: Games

OR/MS Index 1978 subject classification: Primary: 234 Games/group decisions/cooperative

\footnotetext{
*Reprinted from Mathematics of Operations Research, 11(1), 19-29, February 1986.

${ }^{\dagger}$ Institut de Recherches Mathématiques Avancées, Université Louis Pasteur

${ }^{\ddagger}$ Center for Operations Research and Econometrics, Louvain-la-Neuve

${ }^{\S}$ Université des Sciences Sociales de Toulouse and GREQE.
} 


\section{Introduction}

The purpose of this paper is to clarify some issues concerning the core as a solution concept for games in coalitional form, as well as the representation of these games by economies of a certain formal type. Our main interest is in games with side-payments in coalitional form. However, we shall imbed their study in the more general theory of games without side-payments.

It is well known that some games with side-payments may have an empty core. Following Shapley and Shubik [9] one associates to such a game its totally balanced cover, which is a game of the same type having a nonempty core. On the other hand, one may also look at the original side-payment game from a geometrical point of view and consider it as some particular (hyperplane) game without side-payments. This in turn allows one to use the notion of totally balanced cover of a game without side-payments as introduced by Billera and Bixby [4]. Hence one is actually lead to contrast two types of "covering" for a side-payment game: namely the totally balanced cover of its geometrical representation as a game without side-payments - we call it the envelope - and the geometrical no-side-payment representation of its totally balanced side-payment cover, called the geometrical cover (§1). Although these two no-side-payment totally balanced games associated to the same side-payment game may differ, their cores always coincide $(\S 2)$.

There is an "economic interpretation" for such a discrepancy. We consider a modification of the notion of "direct market" of Shapley-Shubik [9] that we call "restricted direct market". We show that the envelope of a side-payment game is the game without side-payments associated to a restricted market, while the geometrical cover is associated to a direct market (§3). The essential difference between these two markets is whether or not a player can obtain utility from a coalition without being a member. Finally (in $\S 4$ ), we compare this new type of market (which is still a pure exchange economy) to the production representation of Billera [3]. In fact we show

that an "input net trade equivalent" of this production representation is the same (up to a trivial identification) as the Rader net trade equivalent of the restricted direct market (see [6]). 


\section{A no-side-payment viewpoint to games with side-payments}

In this section we give the basic definitions and notation. For a set $N=\{1,2, \cdots, i, \cdots, n\}$ of "players" we consider games with side-payments in coalitional form as real-valued functions $v$ defined on $P(N)$, the set of all nonempty subsets of $N$, called "coalitions". To simplify and unify their economic representation we shall consider only 0-normalized games, i.e. games $v$ such that $\forall i \in N, v(\{i\})=0$, and impose the weak monotonicity condition that: $\forall S \in P(N), v(S) \geq 0$.

For any game $v$ the core of $v$ is the set

$$
\operatorname{Core}(v) \stackrel{\text { def }}{=}\left\{u \in \mathbb{R}^{N} ;\left\langle u ; \mathbb{1}_{N}\right\rangle=v(N), \forall S \in P(N),\left\langle u, \mathbb{I}_{S}\right\rangle \geq v(S)\right\}
$$

where $\mathbb{I}_{S} \in\{0,1\}^{N}$ is the characteristic function ${ }^{1}$ of $S \in P(N)$. This can be interpreted as the set of payoff vectors feasible for the grand coalition which cannot be objected against by other coalitions.

Define for $T \in P(N)$, the set $\mathcal{B}_{T}$ of balanced families of coefficients on $T$ by:

$$
\mathcal{B}_{T} \stackrel{\text { def }}{=}\left\{\beta \in \mathbb{R}_{+}^{P(T)} ; \sum_{S \in P(T)} \beta(S) \mathbb{I}_{S}=\mathbb{I}_{T}\right\} .
$$

To a game $v$, we associate, as in Shapley-Shubik [9], the game $\bar{v}$, called the totally balanced cover of $v$ which is:

$$
\bar{v}(T) \stackrel{\text { def }}{=} \sup _{\gamma \in \mathcal{B}_{T}} \sum_{S \in P(T)} \gamma(S) v(S), \quad T \in P(N) .
$$

The game $v$ is said to be balanced if and only if $\bar{v}(N)=v(N)$, and totally balanced if and only if $\bar{v}=v$.

Define the sets $A \stackrel{\text { def }}{=}\left\{u \in \mathbb{R}^{N} ; \forall S \in P(N),\left\langle u, \mathbb{I}_{S}\right\rangle \geq v(S)\right\}$, and $B \stackrel{\text { def }}{=}\left\{u \in \mathbb{R}^{N} ; \forall S \in\right.$ $\left.P(N),\left\langle u, \mathbb{I}_{S}\right\rangle \geq \bar{v}(S)\right\}$. We have $A=B$. The result follows from the inequality $\bar{v} \geq v$ (see (1.2)) and from the fact that for each $T \in P(N)$ and $\delta \in \mathcal{B}_{T}$ such that $\bar{v}(T)=\sum_{S \in P(T)} \delta(S) v(S)$, we clearly have (see (1.1)): $\left\langle u, \mathbb{I}_{T}\right\rangle=\sum_{S \in P(T)} \delta(S)\left\langle u, \mathbb{I}_{S}\right\rangle$. Consider now the two following

\footnotetext{
${ }^{1}$ For any $S \in P(N)$, we have $\mathbb{I}_{S}(i)=1$, if $i \in S$, and $\mathbb{I}_{S}(i)=0$ otherwise. Vectors in $\mathbb{R}^{N}$ are called "payoff vectors" and $\left\langle u, \mathbb{I}_{S}\right\rangle=\sum_{i \in S} u_{i}$ may be interpreted as the "total amount obtained by the coalition" S.
} 
programs:

$$
\begin{aligned}
& \min _{u \in A}\left\langle u, \mathbb{I}_{N}\right\rangle \text { and } \\
& \min _{u \in B}\left\langle u, \mathbb{I}_{N}\right\rangle .
\end{aligned}
$$

By looking at the dual of (1.3), one finds that each optimal solution $u$ of (1.3) fulfills $\left\langle u, \mathbb{I}_{N}\right\rangle=$ $\bar{v}(N)$. Therefore the optimal solutions of (1.4), as well as the optimal solutions of (1.3), are exactly the elements ${ }^{2}$ of $\operatorname{Core}(\bar{v})$. Moreover, we have $\operatorname{Core}(v) \neq \emptyset$ if and only if $v$ is balanced, which is the well-known Bondareva-Shapley Theorem (see [8]).

A more general class of games in coalitional form for a set $N$ of players is the class of games without side-payments. Let $\mathbb{R}^{S} \stackrel{\text { def }}{=}\left\{u \in \mathbb{R}^{N} ; \forall i \notin S, u_{i}=0\right\}$ and $\mathbb{R}_{+}^{S} \stackrel{\text { def }}{=}\left\{u \in \mathbb{R}^{S} ; \forall i \in S, u_{i} \geq\right.$ $0\}$. A game without side-payments is a correspondence $V$ from $P(N)$ to $\mathbb{R}^{N}$ such that, for each $S \in P(N), V(S)$ is nonempty, comprehensive (i.e. $V(S)=V(S)-\mathbb{R}_{+}^{S}$ ) and, for each $a^{S} \in V(S)$, $V(S) \cap\left(\left\{a^{S}\right\}+\mathbb{R}_{+}^{S}\right)$ is compact. In connection to the market representation of games without side-payments we shall only consider games which are compactly generated ${ }^{3}$ namely games $V$ such that, $\forall S \in P(N), V(S)=C_{S}-\mathbb{R}_{+}^{S}$, where $C_{S}$ is a nonempty compact subset of $\mathbb{R}^{S}$.

The Core of a game $V$ without side-payments is the set:

$$
\text { Core }(V) \stackrel{\text { def }}{=}\left\{u \in V(N): \forall S \in P(N), \nexists u^{\prime} \in V(S) \mid \forall i \in S, u_{i}^{\prime}>u_{i}\right\} .
$$

Billera [3] defines (see his definition $(3.1))^{4}$ the totally balanced cover $\bar{V}$ of a game without side-payments $V$ by:

$$
\bar{V}(T) \stackrel{\text { def }}{=} \bigcup_{\delta \in \mathcal{B}_{T}} \sum_{S \in P(T)} \delta(S) V(S), \quad T \in P(N) .
$$

A game $V$ is said to be totally balanced if and only if $\bar{V}=V$. Such a game has a nonempty core (see $[7])$.

\footnotetext{
${ }^{2}$ To each game $v$, one may associate the game $v^{*}$, called the balanced cover of $v$ and defined by: $v^{*}(N)=\bar{v}(N)$, and $\forall S \neq N, v^{*}(S)=v(S)$. Clearly by the same argument we have $\operatorname{Core}\left(v^{*}\right)=\operatorname{Core}(\bar{v})$.

${ }^{3}$ This terminology is due to Billera [3].

${ }^{4}$ The notion was also discussed in Baudier [2] and originally used by Scarf in the prepublication version of [7]. As mentioned in Billera and Bixby [4] it is stronger than the notion of Scarf [7], which makes no use of the summation operator. Since we will be dealing exclusively with games without side-payments derived from games with side-payments, it seems that most objections against using such an operator vanish.
} 
We define the geometrical representation of a game with side-payments $v$ as the game without side-payments $H_{+}(v)$ given by ${ }^{5}$

$$
H_{+}(v)(T) \stackrel{\text { def }}{=}\left\{u \in \mathbb{R}_{+}^{T} ;\left\langle u, \mathbb{I}_{T}\right\rangle \leq v(T)\right\}-\mathbb{R}_{+}^{T}, \quad T \in P(N) .
$$

For any side-payment game $v$, the totally balanced cover $\bar{H}_{+}(v) \stackrel{\text { def }}{=} \overline{H_{+}(v)}$ of the geometrical representation $H_{+}(v)$ of $v$ is called, for short, the envelope of $v$. On the other hand, the geometrical representation $H_{+}(\bar{v})$ of the totally balanced cover $\bar{v}$ of $v$ is called, for short, the geometrical cover of $v$. It is quite natural to compare, for a given game with side-payments $v$, the two alternative ways to compose the geometrical representation operator with the totally balanced cover operator, giving respectively $\bar{H}_{+}(v)$ and $H_{+}(\bar{v})$. We first have:

$$
\text { for each game } v, \bar{H}_{+}(v) \subset H_{+}(\bar{v}) \text {. }
$$

This first result follows from two easy observations, namely: $\bar{H}_{+}(v) \subset \bar{H}_{+}(\bar{v})$ and $\bar{H}_{+}(\bar{v})=$ $H_{+}(\bar{v})$.

\section{Envelopes and geometrical covers of side-payment games: a comparison}

The purpose of this section is to push further the comparison between the two different types of games without side-payments having a nonempty core - envelopes and geometrical covers which we have associated to games with side-payments. The first result demonstrates that for any side-payment game $v$, satisfying an additional monotonicity condition but being not totally balanced, the envelope $\bar{H}_{+}(v)$ effectively differs from the geometrical cover $H_{+}(\bar{v})$.

\footnotetext{
${ }^{5}$ An alternative representation of $v$ is its hyperplane representation defined by

$$
H(v)(S)=\left\{u \in \mathbb{R}^{S} ;\left\langle u, \mathbb{I}_{S}\right\rangle \leq v(S)\right\}, \quad S \in P(N)
$$
}

This definition results in a game without side-payments but is not compactly generated. 
Theorem 2.1 ${ }^{6}$ For a game with side-payments $v$, if, for some $T \subset N$, $\sup _{S \in P(T)} v(S)=$ $v(T)<\bar{v}(T)$ then $\bar{H}_{+}(v)(T) \underset{\neq}{\subset} H_{+}(\bar{v})(T)$.

Proof. It is enough to prove the result for $T=N$. We already know (see $(1.7))$ that $\bar{H}_{+}(v)(N) \subset$ $H_{+}(\bar{v})(N)$. Take a payoff vector $\bar{v}(N) \mathbb{1}_{\{i\}}$ which clearly belongs to $H_{+}(\bar{v})(N)$. Assume $\sup _{S \in P(N)} v(S)=v(N)<\bar{v}(N)$ : we have $\bar{v}(N) \mathbb{1}_{\{i\}} \notin \bar{H}_{+}(v)(N)$. Otherwise we could find $\delta \in \mathcal{B}_{N}$ and $x^{S} \in H_{+}(v)(S) \cap \mathbb{R}_{+}^{S}, S \in P(N)$, such that:

$$
\bar{v}(N)=\sum_{\substack{\delta(S)>0 \\ S \ni i}} \delta(S) x_{i}^{S} \leq \sum_{\substack{\delta(S)>0 \\ S \ni i}} \delta(S) v(S) \leq v(N) .
$$

This is a contradiction.

Although Theorem (2.1) requires a monotonicity assumption, it shows that the geometrical cover and the envelope are different for a very large class of games with side-payments. On the other hand, we shall now prove that the core concept does not discriminate between geometrical covers and envelopes.

Theorem 2.2 ${ }^{7} \operatorname{Core}\left(\bar{H}_{+}(v)\right)=\operatorname{Core}\left(H_{+}(\bar{v})\right)=\operatorname{Core}(\bar{v})$.

\section{Proof.}

1. For each $u \in \mathbb{R}^{n}$ and $\gamma \in \mathcal{B}_{N}$, we have $u=\sum_{S \in P(N)} \gamma(S) u^{S}$, (where $u^{S}$ is the projection of $u$ on $\left.\mathbb{R}^{S}\right)$. If we assume that $u \in \operatorname{Core}\left(H_{+}(\bar{v})\right)$, and $\bar{v}(N)=\sum_{S \in P(N)} \gamma(S) v(S)$, we find that $u$ is an optimal solution of the problems (1.4) and (1.3), while $\gamma$ is an optimal solution of the dual of (1.3). Therefore, whenever $\gamma(S)>0$, we have $\left\langle u^{S}, \mathbb{1}_{S}\right\rangle=v(S)$, which means that $u=\sum_{S \in P(N)} \gamma(S) u^{S}$ is a feasible payoff vector in the game $\bar{H}_{+}(v)$. Moreover, for any $T \subset N$, the inequality $\left\langle u, \mathbb{I}_{T}\right\rangle \geq \bar{v}(T)$ implies that, for any $x \in \bar{H}_{+}(v)(T) \subset H_{+}(\bar{v})(T)$, one cannot have: $\forall i \in T, x_{i}>u_{i}$. Hence $\operatorname{Core}\left(H_{+}(\bar{v})\right) \subset \operatorname{Core}\left(\bar{H}_{+}(v)\right)$.

\footnotetext{
${ }^{6}$ An analogous result holds for hyperplane representations. Formally, if $v$ satisfies $\sup _{S \in P(T)} v(S)=v(T)<$ $\bar{v}(T)$, for some $T \subset N$, then $\bar{H}(v)(t) \underset{\neq}{\subset} H(\bar{v})(T)$.

${ }^{7}$ Here also an analogous result holds for hyperplane representations: $\operatorname{Core}(\bar{H}(v))=\operatorname{Core}(H(\bar{v}))=\operatorname{Core}(\bar{v})$.
} 
2. Conversely, we suppose $u \in \operatorname{Core}\left(\bar{H}_{+}(v)\right)$. We already know that $u \in H_{+}(\bar{v})(N)$ and so $\left\langle u, \mathbb{I}_{N}\right\rangle \leq \bar{v}(N)$. If, for some $T$, we had $\left\langle u, \mathbb{I}_{T}\right\rangle\left\langle v(T)\right.$, the vector: $x=v(T) u^{T} /\left\langle u, \mathbb{I}_{T}\right\rangle$ (or $v(T) \mathbb{I}_{T} /|T|$ if $\left\langle u, \mathbb{I}_{T}\right\rangle=0$ ) would be feasible in $\bar{H}_{+}(v)(T)$. Moreover, we would have: $\forall i \in T, x_{i}>u_{i}$. This is a contradiction. Therefore, $u$ is an optimal solution of the program (1.3), hence of (1.4): $u \in$ Core $H_{+}(\bar{v})$.

From this second result we see that, as far as the Core solution concept is concerned, envelopes and geometrical covers cannot be differentiated. To enhance this difference we shall now compare them from another point of view, the one provided by market games representations.

\section{Envelopes of side-payment games and their restricted market representations}

Since Shapley and Shubik [9], the literature on market games has provided various representations of totally balanced games, with or without side-payments, as "market games" - games that are derived from some type of economy.

In the preceding sections we associated to any game $v$ two different totally balanced games without side-payments, namely the geometrical cover $H_{+}(\bar{v})$ and the envelope $\bar{H}_{+}(v)$. We shall look in this section for two simple types of economies from which these two totally balanced games can be respectively derived. These must be in general distinct (Theorem 2.1), but should be as related as possible (Theorem 2.2), in order to capture the nature of the discrepancy between $H_{+}(\bar{v})$ and $\bar{H}_{+}(v)$. The direct markets in the sense of Shapley and Shubik [9] and a modification of them, the restricted direct markets, will provide the desired result.

Let us first recall some notions about market games. An n-agent, m-commodity simple market is an exchange economy of a special kind denoted: $\mathcal{E}=\left\{\left(Z^{i}, w^{i}, U^{i}\right) ; i \in N\right\}$, where each agent $i \in N$ has a consumption set $Z^{i}=[0,1]^{m}$, a continuous, concave, monotone increasing utility function $U^{i}:[0,1]^{m} \rightarrow \mathbb{R}_{+}$, and initial endowments $w^{i} \in[0,1]^{m}$, with $\sum_{i \in N} w^{i} \leq(1,1, \cdots, 1)$. 
The set of feasible allocations ${ }^{8}$ for a coalition $S \in P(N)$ is

$$
Z^{S} \stackrel{\text { def }}{=}\left\{z^{S}=\left(z^{i}\right)_{i \in S} ; z^{i} \in Z^{i}, \sum_{i \in S} z^{i}=\sum_{i \in S} w^{i}\right\}
$$

The associated market game with side-payments is denoted $v_{\mathcal{E}}$ and defined by:

$$
v_{\mathcal{E}}(S) \stackrel{\text { def }}{=} \sup _{z^{S} \in Z^{s}} \sum_{i \in S} U^{i}\left(z^{i}\right), \quad S \in P(N) .
$$

Since the utility functions $U^{i}$ are nonnegative, the game $v_{\mathcal{E}}$ is nonnegative valued. One may also associate to the simple market $\mathcal{E}$ a game without side-payments which will be denoted $\mathcal{V}_{\mathcal{E}}$ :

$$
\nu_{\mathcal{E}}(S) \stackrel{\text { def }}{=}\left\{u \in \mathbb{R}_{+}^{S} ; \exists z^{S} \in Z^{S} \mid \forall i \in S, U^{i}\left(z^{i}\right) \geq u_{i}\right\}, \quad S \in P(N) .
$$

In the case where all $U^{i}$ are equal to the same concave positively homogeneous of degree 1 utility function $U$, Shapley and Shubik $[9,(3.3)]$ prove $^{9}$ that $v_{\mathcal{E}}(S)=U\left(\mathbb{I}_{S}\right)$. Since $\mathcal{V}_{\mathcal{E}}(S) \cap \mathbb{R}_{+}^{S}$ is convex, contained in $H_{+}\left(v_{\mathcal{E}}\right)(S)$ and contains all vectors $U\left(\mathbb{I}_{S}\right) \mathbb{I}_{\{i\}}=v_{\mathcal{E}}(S) \mathbb{I}_{\{i\}}$, we have:

$$
\nu_{\mathcal{E}}=H_{+}\left(v_{\mathcal{E}}\right)
$$

Define now for $\tau \in[0,1]^{N}$ the set $\mathcal{B}_{\tau} \stackrel{\text { def }}{=}\left\{\beta \in \mathbb{R}_{+}^{P(N)} ; \sum_{S \in P(N)} \beta(S) \mathbb{I}_{S}=\tau\right\}$. Following Shapley and Shubik [9] associate to a game with side-payments $v$ an $n$-agent, $m$-commodity simple market, called the direct market generated by $v$, which we denote $\mathcal{D}_{v}$ :

$$
\mathcal{D}_{v}=\left\{\left(Z^{i}, w^{i}, U\right) i \in N\right\},
$$

\footnotetext{
${ }^{8}$ The justification for using an equality constraint for feasibility comes from the assumption that the utility functions are monotone increasing. In Shapley and Shubik [9] the utility functions are only assumed to be continuous and concave real-valued functions. Hence in some cases the equality constraint for feasibility (which is needed for their results) seems to be less justified from an economic viewpoint.

${ }^{9}$ Because of the equality constraint in the feasible set the only assumptions needed are the ones stated. However see the discussion in footnote 8 .
} 
where, for every $i \in N, Z^{i}=[0,1]^{N}, w^{i}=\mathbb{I}_{\{i\}}$, and ${ }^{10}$

$$
U(\tau)=\sup _{\gamma \in \mathcal{B}_{\tau}} \sum_{S \in P(N)} \gamma(S) v(S)
$$

Shapley and Shubik [9] show that, for any side-payment game $v$,

$$
v_{\mathcal{D}_{v}}=\bar{v}
$$

and that for a totally balanced game $v: v=v_{\mathcal{D}_{v}}$.

Looking at the direct market $\mathcal{D}_{v}$ from a non-side-payment point of view, and combining (3.3) and (3.5), we get:

$$
\mathcal{V}_{\mathcal{D}_{v}}=H_{+}(\bar{v}) .
$$

Hence, from a non-side payment point of view, the direct market does provide us with a representation of the geometrical cover.

To obtain a representation of the envelope $\bar{H}_{+}(v)$, let us reconsider the classical interpretation of goods and utilities in $\mathcal{D}_{v}$. In a direct market, each individual initial endowment $w^{i}=\mathbb{I}_{\{i\}}$ may be interpreted as the "total available time of agent $i$ ". Given any allocation $\left(\tau^{1}, \cdots, \tau^{n}\right)$ feasible for the grand coalition, that is, for every $i \in N, \tau^{i} \in[0,1]^{N}$ and $\sum_{i \in N} \tau^{i}=\mathbb{1}_{N}$, we may also interpret every $\tau_{j}^{i}$ as the "amount of time agent $j$ is at the disposal of agent $i$ ". Finally, considering some $\gamma^{i} \in \mathcal{B}_{\tau^{i}}, \gamma^{i}(S)$ may be interpreted as the "time coalition $S$ meets for agent $i$ " and $\gamma^{i}(S) v(S)$ as the return which agent $i$ receives in the game $v$ for the assembly of $S$. Thus, the utility of agent $i$, which is given by

$$
U\left(\tau^{i}\right)=\sum_{S \in P(N)} \gamma^{i}(S) v(S), \text { for some } \gamma^{i} \in \mathcal{B}_{\tau^{i}}
$$

measures the "total return" obtained by player $i$ in the game, assuming that he is allowed to benefit also from meetings in which he does not participate.

\footnotetext{
${ }^{10}$ This function $U$ is positively homogeneous of degree one and super-additive (hence concave) on $[0,1]^{N}$. A separation theorem ensures that the super-differential $\partial(U)\left(\mathbb{I}_{N}\right)$ of $U$, extended to $\mathbb{R}_{+}^{N}$ and taken as $\mathbb{I}_{N}$, is nonempty. With $\mathcal{B}_{\mathbb{I}_{S}}$ being identified to $\mathcal{B}_{S}$, the restriction of $U$ to $\{0,1\}^{N}$ is $\bar{v}$ and we have $\partial(U)\left(\mathbb{I}_{N}\right)=$ Core $(\bar{v})$. See Aubin [1].
} 
Let us now assume that the meeting programs for agent $i$ are restricted only to those assemblies (coalitions) of which he is a member, i.e. $\{S \in P(N) ; i \in S\}$. This restriction will prove to be sufficient to get a market representation of $\bar{H}_{+}(v)$. We call restricted direct market associated to the game $v$ the $n$-agent, $n$-good simple market denoted $\mathcal{R}_{v}$ and defined as:

$$
\mathcal{R}_{v}=\left\{\left(Z^{i}, w^{i}, U^{i}\right) ; i \in N\right\}
$$

where, for every $i \in N, Z^{i}=[0,1]^{N}, w^{i}=\mathbb{I}_{\{i\}}$,

$$
U^{i}(\tau)=\sup _{\gamma \in \mathcal{B}_{\tau}}\left(\sum_{\substack{S \in P(N) \\ S \ni i}} \gamma(S) v(S)\right) .
$$

Since $v \geq 0$, we note that $U \geq U^{i}$, for every $i \in N$.

As wished, the economy $\mathcal{R}_{v}$ is very similar to $\mathcal{D}_{v}$, while providing a representation of $\bar{H}_{+}(v)$.

Theorem 3.3 The game without side-payments $\mathcal{V}_{\mathcal{R}_{v}}$, associated to the restricted direct market of the game $v$, is the envelope $\bar{H}_{+}(v)$ of $v$.

\section{Proof.}

1. Consider, for some $T \in P(N)$, some $\bar{x} \in \bar{H}_{+}(v)(T) \cap \mathbb{R}_{+}^{T}$. We have $\bar{x}=\sum_{S \in P(T)} \gamma(S) \bar{x}^{S}$, with $\gamma \in \mathcal{B}_{T}$ and $\bar{x}^{S} \in H_{+}(v)(S)\left(\right.$ or $\left.\left\langle\bar{x}^{S}, \mathbb{1}_{S}\right\rangle \leq v(S)\right)$. Letting $x^{S}=v(S) \mathbb{1}_{S} /|S|$ if $\bar{x}^{S}=0$ and $x^{S}=v(S) \bar{x}^{S} /\left\langle\bar{x}^{S}, \mathbb{I}_{S}\right\rangle$ otherwise, it is easy to see that $\bar{x} \leq x \stackrel{\operatorname{def}}{=} \sum_{S \in P(T)} \gamma(S) x^{S}$.

Let us then define, for $i \in T, \gamma^{i}(S) \geq 0$ by:

$$
\begin{array}{ll}
\gamma^{i}(S)=0 & \text { if } i \notin S, \\
\gamma^{i}(S)=\frac{1}{|S|} \gamma(S) & \text { if } i \in S \text { and } v(S)=0, \\
\gamma^{i}(S)=\frac{\gamma(S)}{v(S)} x_{i}^{S} & \text { if } i \in S \text { and } v(S) \neq 0 .
\end{array}
$$

Then, for each $S, \sum_{i \in S} \gamma^{i}(S)=\gamma(S)$, and for each $j \in T, \sum_{S \subset T ; S \ni j}\left(\sum_{i \in S} \gamma^{i}(S)\right)=1$ since $\gamma \in \mathcal{B}_{T}$. 
Defining also $\tau^{i}=\sum_{S \subset T} \gamma^{i}(S) \mathbb{I}_{S}$ for $i \in T$, we find that

$$
\begin{aligned}
& \sum_{i \in T} \tau^{i}=\sum_{i \in T} \sum_{S \subset T} \gamma^{i}(S) \mathbb{I}_{S} \\
& =\sum_{i \in T} \sum_{\substack{S \subset T \\
S \ni i}} \gamma^{i}(S) \sum_{j \in S} \mathbb{I}_{\{j\}} \\
& =\sum_{i \in T} \sum_{j \in T} \sum_{\substack{S \subset T \\
S \ni i \\
S \ni j}} \gamma^{i}(S) \mathbb{I}_{\{j\}} \\
& =\sum_{j \in T} \mathbb{I}_{\{j\}} \sum_{i \in T} \sum_{\substack{S \subset T \\
S \ni i}} \gamma^{i}(S) \\
& =\sum_{j \in T} \mathbb{I}_{\{j\}} \sum_{\substack{S \subset T \\
S \ni j}} \sum_{i \in S}^{\substack{S \ni j \\
S}} \gamma^{i}(S) \\
& =\mathbb{I}_{T}
\end{aligned}
$$

which means that $\left(\tau^{i}\right)_{i \in T}$ is an allocation in the restriction of $T$ of $\mathcal{R}_{v}$. The decomposition defining $\tau^{i}$ shows that:

$$
U^{i}\left(\tau^{i}\right) \geq \sum_{\substack{S \subset T \\ S \ni i}} \gamma^{i}(S) v(S)=\sum_{\substack{S \subset T \\ S \ni i \\ v(S)>0}} \gamma^{i}(S) v(S)
$$

while:

$$
x_{i}=\sum_{\substack{S \subset T \\ S \ni i}} \gamma(S) x_{i}^{S}=\sum_{\substack{S \subset T \\ S \ni i \\ v(S)>0}} \gamma(S) x_{i}^{S}=\sum_{\substack{S \subset T \\ S \ni i \\ v(S)>0}} \gamma^{i}(S) v(S) .
$$

We have proved that $\bar{x}_{i} \leq x_{i} \leq U^{i}\left(\tau^{i}\right), i \in T$, that is $\bar{H}_{+}(v)(T) \subset \mathcal{V}_{\mathcal{R}_{v}}(T)$, for each $T \in P(N)$.

2. To prove the inclusion $\mathcal{V}_{\mathcal{R}_{v}} \subset \bar{H}_{+}(v)$, it is sufficient to prove, for each decomposition $\mathbb{1}_{T}=\sum_{i \in T} \tau^{i}, \tau^{i} \in[0,1]^{n}$, and each decomposition $\tau^{i}=\sum_{S \subset T} \gamma^{i}(S) \mathbb{I}_{S}$, that the vector $u$ of all $u_{i}=\sum_{S \ni i} \gamma^{i}(S) v(S)$, for $i \in T, u_{i}=0$ for $i \notin T$, is equal to or dominated by some 
vector of $\bar{H}_{+}(v)(T)$. For $S \subset T$ we define $x^{S}$ as 0 if $\sum_{k \in T} \gamma^{k}(S)=0$, and otherwise by:

$$
\begin{array}{ll}
x_{i}^{S}=0 & \text { for } i \notin S, \\
x_{i}^{S}=\frac{\gamma^{i}(S) v(S)}{\sum_{k \in T} \gamma^{k}(S)} & \text { for } i \in S .
\end{array}
$$

We find that $x^{S}=\left(x_{i}^{S}\right)_{i}$ belongs to $H_{+}(v)(S)$. Then, defining $\gamma(S) \stackrel{\text { def }}{=} \sum_{k \in T} \gamma^{k}(S)$, we find that $\gamma \in \mathcal{B}_{T}$,

$$
u=\sum_{\substack{S \subset T \\ \gamma(S)>0}} \sum_{i \in S} \gamma^{i}(S) v(S) \mathbb{I}_{\{i\}}=\sum_{S \subset T} \gamma(S) x^{S} \in \bar{H}_{+}(v)(T)
$$

Hence Theorem 3.3 establishes, for a game with side-payments $v$, the same relationship between the restricted direct market $\mathcal{R}_{v}$ and the envelope $\bar{H}_{+}(v)$ as the one that can be established (from Shapley and Shubik [9]) between the direct market $\mathcal{D}_{v}$ and the geometrical cover $H_{+}(\bar{v})$.

Finally, the analogy can be pushed a step further and the relationship between the core of the game $\bar{H}_{+}(v)$ and the competitive payoff in the restricted market $\mathcal{R}_{v}$ can be examined. There is, for the restricted direct market $\mathcal{R}_{v}$, a result similar to Theorem 1 in Shapley-Shubik [10] which states: "every payoff vector in the Core of a totally balanced game is competitive in the direct market of that game". To be more precise, given a Core allocation $\tau=\left(\tau^{1}, \cdots, \tau^{2}, \cdots, \tau^{n}\right)$ in the economy $\mathcal{R}_{v}$, define $p=\left(u^{i}\left(\tau^{i}\right)\right)_{i \in N} \in \mathbb{R}_{+}^{N}$; it may be verified that the Core allocation $\tau$ is competitive both in $\mathcal{R}_{v}$ and in $\mathcal{D}_{v}$ with respect to the "price vector" $p$. Since $p \in \operatorname{Core} \bar{H}_{+}(v)$, we must have $\left\langle p, \mathbb{I}_{S}\right\rangle \geq v(S)$, for each $S \subset N$. For each $t \in[0,1]^{N}$ and each decomposition $\tau=\sum_{S \in P(N)} \gamma(S) \mathbb{I}_{S}$, we therefore find that $\langle p, \tau\rangle \geq \sum_{S \in P(N)} \gamma(S) v(S)$. That is we get the inequality $\langle p, \cdot\rangle \geq U(\cdot)$, between functions on $[0,1]^{N}$. The inequalities $U \geq U^{i}$, together with the equality $\sum_{i \in N}\left\langle p, \tau^{i}\right\rangle=\left\langle p, \mathbb{1}_{N}\right\rangle$, finally proves that, for every $i \in N,\left\langle p, \tau^{i}\right\rangle=U^{i}\left(\tau^{i}\right)=U\left(\tau^{i}\right)$.

As a corollary, we get the inclusion Core $\bar{H}_{+}(v) \subset$ Core $H_{+}(\bar{v})$ in Theorem 2.2. But, we also have from part (1) in the proof of Theorem 3.3, that, for each $x \in \operatorname{Core} H_{+}(\bar{v}), x$ is dominated by some vector $\left(U^{i}\left(\tau^{i}\right)\right)_{i \in N}$ ( since $\left.x \in \bar{H}_{+}(v)(N)\right)$. The inequalities $U \geq U^{i}$ ensure 
that $x_{i}=U^{i}\left(\tau^{i}\right)=U\left(\tau^{i}\right)$, which proves the inclusion Core $H_{+}(\bar{v}) \subset$ Core $\bar{H}_{+}(v)$. Therefore, an alternative proof of Theorem 2.2 may be obtained via the representations $\mathcal{D}_{v}$ and $\mathcal{R}_{v}$ of $H_{+}(\bar{v})$ and $\bar{H}_{+}(v)$.

\section{The production representation of the envelope and its net trade equivalent}

We defined in (3.7) the restricted direct market $\mathcal{R}_{v}$, which was shown to provide a representation of the envelope $\bar{H}_{+}(v)$, and an explanation of the discrepancy between the envelope and the geometrical cover $H_{+}(\bar{v})$.

The paper by Billera [3] provides, for a game $V$ without side-payments, a representation of its totally balanced cover $\bar{V}$ (see (1.5)) as the market game of some $n$-agent, $2 n$-good economy $\mathcal{P}_{V}$, with production. The author also mentions that by taking the "net trade Rader equivalent" of $\mathcal{P}_{V}$ one obtains an $n$-agent, $2 n$-good net trade economy with the same market game, that is $\bar{V}$. In this section we define an input net trade equivalent of $\mathcal{P}_{V}$ and prove that, in the special case of $V=H_{+}(v)$, it is the same as the net trade equivalent of the restricted direct market $\mathcal{R}_{v}$. This provides a second view of the envelope $\bar{H}_{+}(v)$.

Let us start with a general game $V$, where, for every $S \in P(N), V(S)=C_{S}-\mathbb{R}_{+}^{S}$, with each $C_{S}$ a compact convex subset of $\mathbb{R}^{S}$ contained in some cube $C=[0, M]^{N}$ of $\mathbb{R}_{+}^{N}$. Notice that in the special case $V=H_{+}(v)$ we simply let $M=\sup _{S \in P(N)} v(S)$. We consider the $n$-agent, $2 n$-good economy with production denoted ${ }^{11} \mathcal{P}_{v}=\left\{\left(\bar{Z}^{i}, \bar{w}^{i}, \bar{U}^{i}, Y^{i}\right), i \in N\right\}$ which is built as follows. Agent's $i \in N$ initial endowment is $\bar{w}^{i}=\left(0, \mathbb{I}_{\{i\}}\right) \in \mathbb{R}^{2 n}$ (later on interpreted as "his time"), his consumption set is $\bar{Z}^{i}=C \times\{0\} \subset \mathbb{R}^{2 n}$, and his utility $\bar{U}^{i}$ is defined by taking the projection on the $i$ th factor in $\mathbb{R}^{2 n}$, i.e. for $\bar{z}^{i}=(c, 0) \in \bar{Z}^{i}, \bar{U}^{i}\left(\bar{z}^{i}\right)=\operatorname{Proj}_{i}(c, 0)=c_{i}$. Every $i \in N$ has also a production possibility set which is the set $Y^{i} \subset \mathbb{R}^{2 n}$ of convex combinations of $2^{n}$ vectors, namely the vector $(0,0)$ and the vectors of the form $\left(c^{S},-\mathbb{1}_{S}\right)$, where fore $S \in P(N)$,

\footnotetext{
${ }^{11}$ See Billera [3, p. 130].
} 
$c^{S} \in C_{S} \subset C$; that is $y^{i} \in Y^{i}$ if and only if

$$
y^{i}=\sum_{S \in P(N)} \alpha_{S}^{i}\left(c^{S},-\mathbb{I}_{S}\right),
$$

where, for every $S \in P(N), \alpha_{S}^{i} \geq 0, \sum_{S \in P(n)} \alpha_{S}^{i} \leq 1$, and $c^{S} \in C_{S} \subset C$. A feasible state of the economy $\mathcal{P}_{v}$ is a family $\left(y^{i}, \bar{z}^{i}\right)_{i \in N}$, with $y^{i} \in Y^{i}, \bar{z}^{i} \in \bar{Z}^{i}$ and

$$
\sum_{i \in N}\left(\bar{z}^{i}-y^{i}\right)=\left(0, \mathbb{I}_{N}\right)=\sum_{i \in N} \bar{w}^{i}
$$

Intuitively speaking we consider the last $n$ goods, the people times, as inputs out of which agent $i$ can produce the first $n$ goods which are the outputs. Agent $i$ only cares about the $i$ th good which can be obtained by using time of people in some coalition $S$ such that $S \ni i$, i.e., to which he belongs. Notice here a striking similarity with the definition of the utility $U^{i}$ in the restricted market $\mathcal{R}_{v}$ (see (3.7)). The restriction on the $\alpha_{S}^{i}$ is related to the fact that in any case agent $i$ can use at most one unit of time of agent $j$. We also see that agent $i$ has no initial endowment of the first $n$ goods (the outputs).

The economy $\mathcal{P}_{V}$ generates a game without side-payments $\mathcal{V}_{\mathcal{P}_{V}}$ in a manner similar to (3.2) (see Billera [3], p. 131). We have:

$$
\begin{aligned}
\mathcal{V}_{\mathcal{P}_{V}}(S)= & \left\{u \in \mathbb{R}^{S}, \forall i \in S, u_{i} \leq \bar{U}^{i}\left(\bar{z}^{i}\right), \bar{z}^{i} \in \bar{Z}^{i}\right. \\
& \left.\sum_{i \in S}\left(\bar{z}^{i}-\bar{w}^{i}\right) \in \sum_{i \in S} Y^{i}\right\}, \quad S \in P(N) .
\end{aligned}
$$

By the argument of Theorem 3.3 in Billera [3] it can be checked that $\mathcal{V}_{\mathcal{P}_{V}}$ is the totally balanced cover $\bar{V}$ of $V$. In particular, for $V=H_{+}(v), \mathcal{V}_{\mathcal{P}_{H+(v)}}$ is the envelope $\bar{H}_{+}(v)$ and, by (3.8), we get:

$$
\mathcal{V}_{\mathcal{P}_{H+(v)}}=\bar{H}_{+}(v)=\mathcal{V}_{\mathcal{R}_{v}} .
$$

Now, while $\mathcal{P}_{H_{+}(v)}$ and $\mathcal{R}$ differ essentially, we shall see how they can be compared by modifying the notion of "net trade equivalent", introduced in Rader [6], and used by Billera [3] and Billera-Bixby [5]. For an economy with production $\mathcal{P}_{V}$ we may define its input net trade equivalent as the set

$$
\mathrm{Eq}\left[\mathcal{P}_{V}\right]=\left\{\left(\bar{Z}_{*}^{i}, \bar{U}_{*}^{i}\right) ; i \in N\right\}
$$


where $\bar{Z}_{*}^{i}$ is agent $i^{\prime} s$ set of admissible net trades, that is $\bar{Z}_{*}^{i}=\{0\} \times\left([0,1]^{N}-\mathbb{1}_{\{i\}}\right)$ and where $\bar{U}_{*}^{i}$, his utility, is defined by letting, for $\bar{z}_{*}^{i}=\left(0, t^{i}\right) \in \bar{Z}_{*}^{i}$,

$$
\bar{U}_{*}^{i}\left(\bar{z}_{*}^{i}\right)=\bar{U}_{*}^{i}\left(0, t^{i}\right)=\sup \bar{U}^{i}\left(y^{i}+\left(0, t^{i}\right)+\left(0, \mathbb{I}_{\{i\}}\right)\right),
$$

the Sup being taken over the set $\left\{y^{i} \in Y^{i} ;\left(y^{i}+\left(0, t^{i}\right)+\left(0, \mathbb{I}_{\{i\}}\right)\right) \in \bar{Z}^{i}\right\}$. To make things clear, denote by $\bar{t}_{k}^{i j}$ the net amount of good $k, 1 \leq k \leq 2 n$, received by agent $i \in N$ from agent $j \in N$ in such an economy. We have by definition:

- $\bar{t}_{k}^{i j}=0$ if $k=1,2, \cdots, n$, and $\bar{t}_{k}^{i i}=0$,

- $\bar{t}_{n+h}^{i j}=0$ if $h \neq i$ and $h \neq j$,

- $-1 \leq \bar{t}_{n+i}^{i j} \leq 0$, with for each $i, \sum_{j \neq i} \bar{t}_{n+i}^{i j} \in[-1,0]$,

- $0 \leq \bar{t}_{n+j}^{i j} \leq 1$, with for each $i, \sum_{j \neq i} \bar{t}_{n+j}^{i j} \in[0,1]$.

Defining $\bar{t}_{k}^{i} \in \mathbb{R}$ as the total net amount of good $k, 1 \leq k \leq 2 n$, received by agent $i$ from all other agents, we get:

- $\bar{t}_{k}^{i}=0$ for $k=1,2, \cdots, n, t_{n+i}^{i} \in[-1,0]$ and

- $\bar{t}_{n+j}^{i} \in[0,1]$ for $i \neq j$.

Therefore we see that the net trade vector $\bar{t}^{i}=\left(\bar{t}_{1}^{i}, \bar{t}_{2}^{i}, \cdots, \bar{t}_{2 n}^{i}\right)$ is of the form $\left(0, t^{i}\right)$, where $t^{i} \in\left([0,1]^{N}-\mathbb{1}_{\{i\}}\right)$ is the net trade vector of the last $n$ goods, and the feasibility constraint is simply $\sum_{i \in N} t^{i}=0$ (in [6] all goods are traded).

The net trade equivalent $\mathrm{Eq}\left[\mathcal{P}_{V}\right]$ generates a game without side-payments, analogously to (3.2) and (4.1), as follows:

$$
\mathcal{V}_{\mathrm{Eq}\left[\mathcal{P}_{V}\right]}(S)=\left\{u \in \mathbb{R}^{S}: \forall i \in S, u_{i} \leq \bar{U}_{*}^{i}\left(\bar{z}_{*}^{i}\right), \bar{z}_{*}^{i} \in \bar{Z}_{*}^{i}, \sum_{i \in S} \bar{z}_{*}^{i}=0\right\} .
$$

On the other hand, an $n$-agent $m$-commodity simple market $\mathcal{E}=\left\{\left(Z^{i}, w^{i}, U^{i}\right), i \in N\right\}$ (as defined in $\S 3$ ) is clearly a special case of a production economy where for every $i \in N, Y^{i}=\{0\}$. 
One can therefore consider the net trade Rader equivalent of $\mathcal{E}$. In particular, given a restricted direct market $\mathcal{R}_{v}$, one obtains the $n$-agent net trade Rader equivalent:

$$
\operatorname{Req}\left[\mathcal{R}_{v}\right]=\left\{\left(T^{i}, U_{*}^{i}\right), i \in N\right\}
$$

where $T^{i}$, $i$ 's set of admissible net trades, is simply $T^{i}=[0,1]^{n}-\mathbb{1}_{\{i\}}$ and $U_{*}^{i}$ is defined by letting, for $t^{i} \in T^{i}$ :

$$
U_{*}^{i}\left(t^{i}\right)=U^{i}\left(t^{i}+\mathbb{1}_{\{i\}}\right)
$$

It is trivial to check that: $\mathcal{V}_{\mathrm{Eq}\left[\mathcal{R}_{v}\right]}=\mathcal{V}_{\mathcal{R}_{v}}$.

Starting from a game with side-payments $v$, consider the two net trade economies Eq $\left[\mathcal{P}_{H_{+}(v)}\right]$ and Req $\left[\mathcal{R}_{v}\right]$. Let $\ell: \mathbb{R}^{2 n} \rightarrow \mathbb{R}^{n}$ be the projection onto the last $n$ coordinates. We have, for every $i \in N, \ell\left(\bar{Z}_{*}^{i}\right)=T^{i}$, where $\bar{Z}_{*}^{i}$ and $T^{i}$ are $i$ 's admissible set of net trades in $\mathrm{Eq}\left[\mathcal{P}_{H+(v)}\right]$ and $\operatorname{Req}\left[\mathcal{R}_{v}\right]$ respectively. The relationship between the net trade equivalent of $\mathcal{P}_{\bar{H}+(v)}$ and the net trade Rader equivalent of $\mathcal{R}_{v}$ results from the following statement:

Theorem 4.1 For every $i \in N$, and every $t^{i} \in[0,1]^{N}-\mathbb{1}_{\{i\}}, \bar{U}_{*}^{i}\left(0, t^{i}\right)=U_{*}^{i}\left(t^{i}\right)$.

Proof. Consider any $i \in N$ and any $t^{i} \in[0,1]^{N}-\mathbb{I}_{\{i\}}=T^{i}$. We have $\left(0, t^{i}\right)=\bar{z}_{*}^{i} \in \bar{Z}_{*}^{i}$. Let us show first: $\bar{U}_{*}^{i}\left(0, t^{i}\right) \leq U_{*}^{i}\left(t^{i}\right)$. Take $y^{i} \in Y^{i},\left(y^{i}+\left(0, t^{i}\right)+\left(0, \mathbb{I}_{\{i\}}\right)\right) \in \bar{Z}^{i}$ such that:

$$
\bar{U}_{*}^{i}\left(0, t^{i}\right)=\bar{U}^{i}\left(y^{i}+\left(0, t^{i}\right)+\left(0, \mathbb{I}_{\{i\}}\right)\right) .
$$

We have: $y^{i}=\sum_{S \in P(N)} \alpha_{S}^{i}\left(C_{S},-\mathbb{I}_{S}\right), \alpha_{S}^{i} \geq 0, \sum_{S \in P(N)} \alpha_{S}^{i} \leq 1$, and $c_{S} \in H_{+}(v)(S) \cap \mathbb{R}_{+}^{S}$. We find:

$$
y^{i}+\left(0, t^{i}\right)+\left(0, \mathbb{1}_{\{i\}}\right)=\left(\sum_{S \in P(N)} \alpha_{S}^{i} c_{S}, 0\right) .
$$

Thus:

$$
\bar{U}_{*}^{i}\left(0, t^{i}\right)=\operatorname{Proj}_{j}\left(\sum_{S \in P(N)} \alpha_{S}^{i} c_{S}, 0\right)=\sum_{S \in P(N)} \alpha_{S}^{i} c_{S}^{i}
$$

and

$$
\sum_{S \in P(N)} \alpha_{S}^{i} \mathbb{I}_{S}=t^{i}+\mathbb{I}_{\{i\}}=\tau^{i} \in[0,1]^{N}
$$


Since we have:

$$
\sum_{S \in P(N)} \alpha_{S}^{i} c_{S}^{i} \leq \sum_{\substack{S \in P(N) \\ S \ni i}} \alpha_{S}^{i} v(S)
$$

we conclude:

$$
\bar{U}_{i}^{*}\left(0, t^{i}\right) \leq U_{*}^{i}\left(t^{i}\right)=\sup _{\gamma \in \mathcal{B}_{\tau^{i}}} \sum_{\substack{S \in P(N) \\ S \ni i}} \gamma(S) v(S), \quad \tau^{i}=t^{i}+\mathbb{I}_{\{i\}} .
$$

Conversely, let $\tau^{i}=t^{i}+\mathbb{1}_{\{i\}} \in[0,1]^{N}$. Given any decomposition

$$
\tau^{i}=\sum_{S \in P(N)} \gamma(S) \mathbb{I}_{S}, \quad \gamma_{S} \geq 0
$$

such that

$$
U_{*}^{i}\left(t^{i}\right)=U^{i}\left(t^{i}+\mathbb{I}_{\{i\}}\right)=\sum_{\substack{S \in P(N) \\ S \ni i}} \gamma(S) v(S)
$$

Let us define:

- $\alpha_{S}^{i}=0$ if $S \not \supset i, \alpha_{S}^{i}=\gamma_{S}$ if $S \ni i$,

- $c_{S}=0$ if $S \not \supset, c_{S}=v(S) \mathbb{1}_{\{i\}}$ if $S \ni i$.

Then we get:

$$
U_{*}^{i}\left(t^{i}\right)=\operatorname{Pr}_{i}\left(\sum_{S \in P(N)} \alpha_{S}^{i} c_{S}, 0\right) .
$$

Let $\bar{z}^{i}=\left(\sum_{S \in P(N)} \alpha_{S}^{i} c_{S}, 0\right) \in \bar{Z}^{i}$ and $y^{i}=\sum_{S \in P(N)} \alpha_{S}^{i}\left(c_{S},-\mathbb{I}_{S}\right) \in Y^{i}$. We have: $\bar{z}^{i}=$ $y^{i}+\left(0, t^{i}\right)+\left(0, \mathbb{I}_{\{i\}}\right)$. Thus, we obtain: $U_{*}^{i}\left(t^{i}\right)=\bar{U}^{i}\left(\bar{z}^{i}\right) \leq \bar{U}_{*}^{i}\left(0, t^{i}\right)$.

Finally using 3.1 in Billera-Bixby [5], (4.2) and (4.3) we get

$$
\bar{H}_{+}(v)=\mathcal{V}_{\mathrm{Eq}\left[\mathcal{P}_{H_{+}(v)}\right]}=\mathcal{V}_{\mathcal{R}_{v}}=\mathcal{V}_{\mathcal{P}_{H_{+}(v)}}=\mathcal{V}_{\operatorname{Req}\left[\mathcal{P}_{H_{+}(v)}\right]} \cdot
$$

\section{References}

[1] Aubin, J.P. (1981). Cooperative fuzzy games. Mathematics of Operations Research, 6(1), 1-13. 
[2] Baudier, E. (1971). Généralisation de la notion de fonction caractéristique dans un jeu. Mimeo, CEPREMAP, Mars 1971.

[3] Billera, L.J. (1974). On games without side payments arising from a general class of markets. Journal of Mathematical Economics, 1, 129-139.

[4] Billera, L.J. and R.E. Bixby (1973). A characterization of polyhedral market games. International Journal of Game Theory, 2, 253-261.

[5] Billera, L.J. and R.E. Bixby (1974). Market representation of $n$-person games. Bulletin of the American Mathematical Society, 80(3), 522-526.

[6] Rader, T. (1964). Edgeworth exchange and general equilibrium. Yale Economic Essays, 14, 133-180.

[7] Scarf, H. (1967). The core of an n-person game. Econometrica, 35, 50-69.

[8] Shapley, L.S. (1967). On balanced sets and cores. Naval Research Logistics Quarterly, 14, $453-460$.

[9] Shapley, L. and M. Shubik (1969). On market games. Journal of Economic Theory, 1, 9-25.

[10] Shapley, L. and M. Shubik (1975). Competitive outcomes in the cores of market games. International Journal of Game Theory, 4, 229-237. 